\title{
Análisis de las estrategias e instrumentos de evaluación en el aprendizaje del inglés como lengua extranjera ${ }^{1}$
}

\author{
Analysis of strategies and instruments of assessment in \\ learning english as a foreign language
}

\author{
Análise de estratégias e instrumentos de avaliação para \\ aprender inglês como língua estrangeira
}

\author{
Maritza Eugenia Ordoñez Delgado \\ Universidad de San Buenaventura \\ Licenciada en educación para la primera infancia \\ Grupo de investigación TAEPE \\ Semillero A.E.I -aprendiendo a evaluar en la infancia \\ maridmdelgado97@hotmail.com \\ Jessica Rodríguez Nomely \\ Universidad de San Buenaventura \\ Licenciada en educación para la primera infancia \\ Grupo de investigación TAEPE \\ Semillero A.E.I -aprendiendo a evaluar en la infancia \\ jessrodrigueznomely@gmail.com
}

\section{Resumen}

Este estudio tiene como finalidad analizar los instrumentos y estrategias de evaluación que utilizaron las maestras en el curso de inglés como lengua extranjera en la primera infancia, a través de una diferenciación entre lo que es la evaluación y la calificación. Lo anterior permite ver cómo cada una de ellas contribuye a un aprendizaje perdurable (evaluación formativa), que trasciende en el aprendizaje de los niños y las niñas, dando sentido al conocimiento y a lo que reconocen. La calificación busca la memorización de un tema, con el fin de obtener una nota; examina, mide y certifica lo que está bien o está mal; ello puede llevar a que el estudiante pierda el interés por el aprendizaje; por esta razón surgió

\footnotetext{
${ }^{1}$ Este artículo es producto del trabajo de grado para optar por el título de Licenciado en educación para la primera Infancia de la Universidad de San Buenaventura - Bogotá, durante el año 2017.
} 
la preocupación por saber si los maestros califican o evalúan, teniendo en cuenta que la evaluación formativa es aquella que tiene en consideración los ritmos de aprendizaje de los niños/as, y permite utilizar diferentes instrumentos ajenos a la calificación que propician la enseñanza de la lengua extranjera.

Palabras claves: aprendizaje del inglés, instrumentos de evaluación, evaluación, evaluación formativa.

\begin{abstract}
The purpose of this study is to analyze the evaluation instruments and strategies used by the teachers in the course of English as a foreign language in early childhood, through a differentiation between what is evaluation and qualification. This allows us to see how each of them contributes to enduring learning (formative evaluation), which transcends the learning of boys and girls, giving meaning to knowledge and what they recognize. The qualification seeks the memorization of a subject, in order to obtain a grade; examines, measures and certifies what is right or wrong; this can lead to the student losing interest in learning; for this reason the concern arose to know if the teachers qualify or evaluate, taking into account that the formative evaluation is one that takes into consideration the learning rhythms of the children, and allows the use of different nonqualifying instruments that favor the teaching of the foreign language.
\end{abstract}

Keywords: English learning, assessment instruments, evaluation, formative evaluation.

\title{
Resumo
}

O objetivo deste estudo é analisar os instrumentos e estratégias de avaliação utilizados pelos professores no curso de inglês como língua estrangeira na primeira infância, através de uma diferenciação entre o que é avaliação e qualificação. Isso nos permite ver como cada um deles contribui para a aprendizagem duradoura (avaliação formativa), que transcende a aprendizagem de meninos e meninas, dando sentido ao conhecimento e ao que eles reconhecem. A qualificação visa a memorização de um assunto, a fim de obter uma nota; examina, mede e certifica o que é certo ou errado; isso pode levar o aluno a perder o interesse em aprender; Por isso, surgiu a preocupação de saber se os professores se qualificam ou avaliam, tendo em vista que a avaliação formativa é aquela que leva em consideração os ritmos de aprendizaje 
Análisis de las estrategias e instrumentos de evaluación en el aprendizaje del inglés como lengua extranjera

das crianças, e permite o uso de diferentes instrumentos não qualificativos que favorecem o aprendizado. ensino da língua estrangeira.

Palavras-chave: aprendizado de inglês, instrumentos de avaliação, avaliação, avaliação formativa.

\section{El aprendizaje de la lengua extranjera en Colombia}

La enseñanza de una lengua extranjera, en este caso el inglés, es importante ya que nos encontramos en un mundo globalizado en el cual es necesario dominar más de una lengua. De acuerdo con lo anterior, en Colombia se da la oportunidad de aprender una lengua extranjera, dándole prioridad al inglés debido a que es considerado como una lengua universal.

Teniendo en cuenta el anterior contexto, esta investigación surge del deseo de conocer las diferentes estrategias e instrumentos de evaluación que se aplican en las dos instituciones a investigar, para evidenciar si realmente los niños; obtienen aprendizajes perdurables o solo memorísticos.

A través de la distinción entre lo que es la evaluación y la calificación, se puede apreciar cómo cada una de ellas contribuye a un aprendizaje perdurable, como en el caso de la evaluación formativa. Pero, por otra parte la calificación conlleva a una memorización de un tema con el fin de obtener una nota o recompensa positiva, pues la estrategia de examinar, calificar medir y certificar recae en que el estudiante reciba una nota a cambio de decir lo que está bien o está mal. Sin embargo, una consecuencia de ese método evaluativo es que el estudiante puede perder el interés por el aprendizaje; por esta razón, surge la preocupación por saber si los maestros califican o evalúan, teniendo en cuenta que la evaluación formativa tiene en consideración los ritmos de aprendizaje de los niños/as, utilizando diferentes instrumentos ajenos a la calificación y como éstos propician la enseñanza de la lengua extranjera.

$\begin{array}{ccr}\text { Por otra parte, } & \text { esta } \\ \text { investigación } & \text { describió } & \text { y }\end{array}$ caracterizó el papel que juegan los instrumentos de evaluación en la formación de la lengua extranjera inglés, para que la evaluación formativa trascienda en el aprendizaje de los niños, dando sentido al conocimiento y a lo que reconoce.

Tomando en cuenta otra perspectiva, es importante en la investigación, observar cuál es la diferencia de la aplicación de éstos instrumentos y estrategias 
de evaluación en dos instituciones que cuentan con contextos y PEI diferentes, vista desde la intensidad de la enseñanza de la lengua extranjera (inglés); hallando similitudes y diferencias entre las acciones de las maestras al evaluar a los niños/as.

\section{Aprendizaje de la lengua extranjera, una perspectiva del estudiante y el maestro}

¿Qué estrategias e instrumentos de evaluación son utilizados en dos instituciones educativas de la ciudad de Bogotá, y cómo estos contribuyen al aprendizaje del inglés como lengua extranjera de los niños y las niñas de 5 a 8 años? fue la pregunta de investigación que orientó el proceso. En este sentido, para las maestras en formación de la universidad de San Buenaventura Bogotá, fue de vital importancia evidenciar las estrategias e instrumentos de evaluación que utilizan las instituciones Gimnasio del Norte y Colegio la Presentación de Fátima, que propician el aprendizaje de la lengua extranjera (Inglés), ya que es necesario trabajar este idioma porque a nivel académico el estudio de las lenguas integra diferentes maneras de hablar, fortaleciendo los niveles cognitivos, permitiéndole a los niños y niñas desde temprana edad desenvolverse en una lengua diferente a la materna, ayudando a estimular el lenguaje abriéndoles caminos al conocimiento de otras culturas. Por lo mismo, los instrumentos de evaluación implementados por las maestras, ayudaron a verificar cuáles servían para identificar el aprendizaje perdurable en los niños, y de qué manera fortalecen y apoyan los procesos de enseñanza-aprendizaje. También permitieron analizar los dos contextos a investigar, hallando diferencias y similitudes en la manera cómo evalúan. Con este tipo de investigación, se pretende analizar la forma de evaluar la lengua extranjera, puesto que Colombia se encuentra entre los países con bajo dominio del inglés ${ }^{2}$, siendo el país con el menor porcentaje en América Latina con el 45,1\% de dominio de la lengua inglesa, según lo establecido por el English Proficiency Índex (2012). De igual manera, se tiene en cuenta que hoy en día el tener conocimiento de una sola lengua no es suficiente en un mundo interconectado.

Por otra parte, para el desarrollo del proyecto, fue importante analizar las relaciones que se establecen entre la lengua nativa y la extranjera, y cómo se evalúa ese proceso de acuerdo con

\footnotetext{
2 El colombiano/ ser bilingüe aporta valor competitivo. Recuperado de http://www.elcolombiano.com/BancoCon ocimiento/S/ser_bilingue_aporta_valor_c ompetitivo/ser_bilingue_aporta_valor_co mpetitivo.asp
} 
Análisis de las estrategias e instrumentos de evaluación en el aprendizaje del inglés como lengua extranjera

el parámetro que se tiene respecto a la evaluación de la lengua materna de cada uno de los niños, en donde tengan la misma importancia la lengua nativa y la extranjera, con base en las necesidades del estudiante(Truscott de Mejía, 2009).

Por último, este estudio permitió evidenciar y analizar la importancia que tiene para un niño o niña la evaluación y el sentido que se le ve a la misma, en este caso se trabajó en torno al inglés como lengua extranjera, pero a través de este también se logra describir el aprendizaje perdurable que tiene un niño a través de las estrategias que utilizan o aplican los maestros en las diferentes actividades; por otra parte como maestras en formación de la Universidad de San Buenaventura, se puede llegar a analizar dichos instrumentos y estrategias de evaluación como medio de experiencia, es decir, este estudio permite que se vea la manera óptima, precisa y motivadora para que el niño y la niña a la hora de generar un aprendizaje perdurable, puedan desempeñarse en el inglés como lengua extranjera, sabiendo que es el aprendizaje que va a desarrollar a lo largo de su vida.

Se demostró a través de entrevistas y observaciones de los procedimientos de evaluación, las estrategias que utilizan las maestras para la enseñanza del inglés, lo cual evidencia la relación maestro-estudiante, los procesos de enseñanza-aprendizaje y los instrumentos de evaluación que potencian dichos aprendizajes.

Por otro lado, se encontró que el inglés apoya a la lengua materna y ayuda al niño a desarrollarse en diferentes áreas del conocimiento como: la matemática, las ciencias sociales, la literatura y las artes.

\section{Metodología}

Esta investigación se realizó bajo el enfoque cualitativo, desde la metodología de análisis de contenidos, ya que permite describir y analizar la complejidad de la realidad social sobre la cual se está investigando, posibilitando a las investigadoras conocer el sentido que tienen los instrumentos de evaluación en estas instituciones. Por medio de los niveles del análisis del contenido (superficie, analítico e interpretativo) se busca llegar a la comprensión, interpretación y a una visión más clara de las estrategias e instrumentos de evaluación que aplican las maestros en la enseñanza de la lengua extranjera en los niños y niñas, siendo este el objeto de investigación, de este modo, se llega a acceder de manera sistemática a dicha información y recolección de datos (Ruiz, s.f, p. 45-61).

El análisis de datos es la
metodología que permite


recopilar, comparar, clasificar y analizar la información observada desde las experiencias en las instituciones, yendo en una línea directa con los objetivos planteados para el proyecto, dándole sentido a los elementos estructurales que determinan las referencias y rangos que propician la recolección de datos nombrada más adelante; siguiendo con una línea creciente, que permite caracterizar las diferentes maneras que tienen las maestras al concebir, aplicar y vivenciar las estrategias e instrumentos de evaluación para propiciar aprendizajes en los niños y las niñas.

Finalmente, la recolección de datos se llevará a cabo en un primer momento, a través de la Identificación junto con la clasificación, entendida como la recopilación y producción de la información a la que se accede, esto, es producto de la interacción del investigador y los informantes, la cual permite alcanzar los niveles descriptivo y analítico; en segundo momento, por medio de la periodización en la cual se ordena la información por medio las etapas o categorías definidas por los investigadores, se pueden hacer notorias las semejanzas y diferencias entre los instrumentos y estrategias de evaluación utilizados por las maestras en las dos instituciones (Gimnasio del Norte y Colegio la Presentación de Fátima) para la enseñanza de la lengua extranjera.

\section{Marco teórico}

\section{La evaluación, campo educativo para contemplar aprendizajes}

La evaluación en general implica en esencia analizar una acción humana, la cual tiene en cuenta al estudiante y su formación; asimismo, posee varias herramientas para usarla en un aula de clase o fuera de ella, las cuales sirven para evidenciar el proceso de aprendizaje; por esta razón nuestro proyecto se centra en el análisis de la evaluación con sus instrumentos y cómo se utilizan en el aula de clase para promover el aprendizaje de una lengua extranjera, en este caso el inglés; teniendo en cuenta que la evaluación debe tener un impacto positivo y promover que los docentes las utilicen para incentivar los procesos de enseñanza-aprendizaje de los niños y niñas y por lo tanto, para conocer la incidencia que tiene la evaluación en la enseñanza del inglés como lengua extranjera.

Teniendo en cuenta lo anterior, la primera categoría que dio base a la investigación, fue el concepto de evaluación propuesto por Álvarez Méndez (2001), el cual es definido como una actividad crítica del aprendizaje porque se asume que gracias a esta se adquiere un mayor conocimiento, se trata de buscar la manera de que sea crítica, puesto que no es igual a calificar, medir, ni tampoco corregir, evidenciando cómo el 
Análisis de las estrategias e instrumentos de evaluación en el aprendizaje del inglés como lengua extranjera

maestro aplica estrategias con un papel instrumental, con el fin de lograr un aprendizaje, este medio debe ser racional, práctico y crítico. Es labor del maestro implementar y generar el gusto en sus estudiantes por una evaluación, sin verla como un castigo, es decir, el niño tiene la libertad de pensar que la realiza no por obtener una calificación o nota a cambio sino porque ésta es esencial en su proceso de aprendizaje. De acuerdo con el autor "el aprendizaje se ejerce esencialmente por voluntad, y de ahí depende el significado que el niño le vea a la evaluación, reconociendo que el objetivo de ésta no es el resultado final sino el proceso que él lleva siguiendo, dándole una oportunidad de seguir aprendiendo a través de ella". De ahí, que el maestro durante mucho tiempo se ha preocupado por la didáctica y el currículo para que el niño aprenda saberes que muchas veces terminan siendo importantes únicamente para un examen, siendo este un aparato de exclusión. Méndez (2001) afirma: "la evaluación se utiliza como un instrumento de exclusión a lo largo del proceso de escolarización mediante procedimientos que seleccionan y marginan, perjudicando las oportunidades educativas posteriores de muchos alumnos". Sin embargo, este no es el objetivo de la educación sino que por el contrario debe incluir y adaptarse a las diferencias de los niños.

\section{Nuevo conocimiento del mundo a través de una lengua}

La segunda categoría que dió base a la investigación y que fue importante conocer, es el concepto de enseñanza, que según (Zuluaga, 2003) se convirtió en una acción práctica donde se aplican los métodos para aprender; es importante reconocer la enseñanza en su función articuladora con otras disciplinas como por ejemplo (enseñanza de las matemáticas, inglés, biología etc.), estableciendo la práctica de la enseñanza como el campo aplicado de la pedagogía en el cual se centra la didáctica.

Así mismo, la enseñanza implica una relación entre la educación y la sociedad; debido a que la enseñanza en una cultura en particular conlleva a una relación entre el conocimiento y la tradición, donde el maestro debe pensar en una enseñanza no sólo práctica, sino como objeto de análisis la cual es capaz de articular una forma de ser del maestro, unos procedimientos, un lugar, unos contenidos y una forma de aprender a lo cual se le denomina didáctica; se entiende por didáctica al discurso a través del cual el saber pedagógico ha pensado la enseñanza hasta hacerla el objeto central de sus elaboraciones.

Teniendo en cuenta lo anterior Zuluaga (2003) también menciona que la enseñanza es la reflexión 
fundamental de la pedagogía ya que ésta no es un simple método, ni un proceso de transmisión de conocimientos sino que posee una naturaleza conceptual y es una práctica de conocimiento, por esta razón se debe entender la enseñanza como aquel espacio que posibilita el pensamiento.

Teniendo en cuenta lo anterior, es primordial también no solo conocer la importancia de la enseñanza, sino también comprender el sentido que se le da y el valor de enseñar a los niños una lengua extranjera, en este caso el inglés. Cabe mencionar que en nuestro proyecto hacemos referencia a lengua extranjera y no segunda 3.

A partir de esto, para enseñar el inglés como lengua extranjera hay que tener en cuenta que esta no solo supone enseñar la gramática, sino también supone enseñar toda una nueva cultura y costumbres diferentes a las propias; hay que tener en cuenta que estamos en un mundo globalizado en el cual es necesario saber más de una lengua; ya que no solo fortalece sus habilidades cognitivas, sino que también como lo menciona Álvarez (2010) "El aprendizaje de inglés como lengua extranjera a edades tempranas

\footnotetext{
3 Lambert (1987): "En Canadá hay dos lenguas nacionales oficiales, y tanto el inglés como el francés pueden ser la primera lengua para un gran número de canadienses."
}

lengua, porque según Lambert (1987) "La distinción comienza a aparecer cuando se piensa en Estados Unidos, donde el inglés es la única lengua nacional y donde, simplificando los términos, el inglés, si no es la lengua familiar de una persona, se espera que pase a ser la «segunda» lengua, mientras que, si el inglés es la lengua que se habla en casa, cualquier otra lengua que se aprenda es «extranjera»"; debido a esto, se tiene en cuenta que nosotros solo tenemos una lengua oficial la cual es el Español, y el inglés se enseña como la lengua extranjera; nosotros como país no tenemos una segunda lengua como en el caso de Canadá

mejora la competencia comunicativa en esa lengua extranjera, pero también en la lengua materna al entender el niño a través del proceso de aprendizaje el lenguaje como un fenómeno y aprender a utilizar las partes que conforman este fenómeno de una forma práctica." por esta razón es importante motivar a los niños/as en el aprendizaje de ésta lengua, "hoy es evidente que el manejo de una sola lengua no es suficiente en un mundo interconectado. $\mathrm{La}$ capacidad de ser bilingüe o multilingüe es cada vez más necesaria para el estudio, el trabajo y la convivencia. Colombia ha tenido una larga tradición de incluir lenguas extranjeras, como el inglés, el francés, el alemán, el italiano en el currículo escolar, 
Análisis de las estrategias e instrumentos de evaluación en el aprendizaje del inglés como lengua extranjera

para que los bachilleres tengan una visión pluralista del mundo y entren en contacto con otras maneras de pensar y de expresarse, a fin de que este reconocimiento de la diversidad lleve a un reconocimiento de la tolerancia y del respeto del otro/otra" (Anne-Marie de Mejía, 2010). De acuerdo con lo anterior, el enseñar la lengua extranjera inglésa favorece los entornos y el desarrollo del niño en los diferentes ambientes y contextos en los que se encuentra, desde lo social, cultural y cognitivo de los niños, además, fomenta el respeto, entendimiento y conciencia de personas en el mundo, teniendo en cuenta su cultura, también, potencia la memoria, el pensamiento-lógico y habilidades comunicativas, generando otras perspectivas acerca del mundo y de lo que lo rodea, teniendo en cuenta la manera de expresarse y cómo se estimula el lenguaje a través del mismo aprendizaje.

\section{La formación como eje transversal}

La formación en nuestro proyecto es una categoría transversal, puesto que a través de ésta podemos conocer cómo influye la evaluación en la enseñanza de una lengua extranjera. Se tiene en cuenta la formación como un proceso que se logra ver desde el momento en el que el niño nace, tomando en cuenta la disciplina como principio fundamental de ésta, la cual lleva a los niños/as a entender el todo; la sociedad, su familia y su entorno, lo cual cabe mencionarlo para esta investigación, ya que es necesario que los niños/as comprendan y vean el sentido a la enseñanza, en este caso del inglés como lengua extranjera; es importante también ver dicho proceso el cual evidencia que tan acertado sea el aprendizaje o lo que se hace a través de la memoria, ya que muchas veces este se produce a corto plazo y no es perdurable; por esta razón, a través de la formación se crean hábitos en los niños y las niñas, en los cuales él actúa por máximas ${ }^{8}$, donde no espere nada a cambio; solo los aprendizajes que quiera obtener y todo esto reunido bajo una formación moral, la cual facilita los procesos de enseñanzaaprendizaje; por ende la cultura moral debe fundamentarse en las máximas, tanto para el maestro como para el estudiante; la evaluación debe tener en cuenta la formación del niño/a, además de la disciplina la cultura y la moral, sin necesidad que la disciplina sea conductista; ya que la conducta moral del niño va acorde con sus principios éticos (Kant, 1986).

Kant menciona la formación

\footnotetext{
${ }^{8}$ Según Kant (1986) en su libro Pedagogía se refiere a actuar por máximas cuando una persona actúa por principios, desde su propia moral sin esperar algo a cambio.
} 
desde un espíritu fuerte para el cual el hombre necesita rehusarse a la satisfacción de sus propios deseos, pues de lo contrario se acostumbrara a cumplir sus inclinaciones y no será capaz de seguir instrucciones ni inhibirse de algo cuando es debido. Por esta razón, el maestro tiene que llegar a pensar que la evaluación va dirigida a la formación del niño/a, además de la disciplina la cultura y la moral, sin necesidad que la disciplina sea conductista; ya que la conducta moral del niño/a va acorde con sus principios éticos; por esta razón Kant y Locke (1987) mencionan la importancia de la autorregulación y que el niño/a se domine a sí mismo, para que pueda reflexionar acerca de su propia educación y así dé a conocer lo que realmente está aprendiendo.

A través del análisis de contenidos que se realizó en las instituciones se pudo evidenciar las diferentes estrategias e instrumentos de evaluación que allí utilizaban para evaluar y observar qué tanto les servía a los niños/as para conocer y aprender de una lengua extranjera inglés.

En primera medida, se comenzó a analizar lo que se observó y vivenció en la institución número uno, la cual cuenta con una serie de características diferenciales a la otra institución. Desde lo que aporta el texto Instrumentos de evaluación para promover el aprendizaje, propuesto por Dorothy Spiller (2011) se caracterizaron los instrumentos usados en la institución y de qué manera los aplican. Según los documentos formales analizados se pudo dar cuenta que muchos de ellos tienen concordancia con lo que se observó, pues los lineamientos de Programa de la Escuela Primaria, describe que debe tener una constante evolución, trabajando con los aprendizajes pertinentes $y$ significativos, centrándose en el desarrollo integral del niño/a, basado en despertar el entusiasmo por aprender y vivir en un mundo interconectado, formando personas para la vida.

Por un lado se encontró que los exámenes eran aplicados en las dos instituciones, con ayuda de un libro abierto o cerrado para evaluar lo siguiente: conocimiento, comprensión, análisis, aplicación, resolución de problemas y visión general (dependiendo del formato del examen), son usados por las maestras al final del periodo para evaluar y medir también qué tanto aprendió el niño/a, este instrumento recae en la calificación cuantitativa que permite que el estudiante memorice la mayor cantidad de temas con el fin de aprobar la materia; en lo observado durante el proceso de investigación se pudo dar cuenta que los niños y las niñas temen un poco al presentar dicho examen, debido a que constantemente los presionan 
Análisis de las estrategias e instrumentos de evaluación en el aprendizaje del inglés como lengua extranjera

para que aprueben con una buena nota; en dicha prueba, además, las preguntas que incluyen en este apartado, suelen ser temas puntuales de los talleres transversales que se manejan en la institución. Sin embargo, se realizan este tipos de exámenes para dar prueba a los padres de familia sobre los aprendizajes de los niños, cabe recalcar que la mayoría de estos son propuestos desde la lengua extranjera inglés, aunque no en todos los cursos y grados se inicia este proceso desde el grado jardín, debido a que a su edad tienen un proceso de lecto-escritura ya adquirido y una expresión oral que les permite diferenciar con facilidad una lengua de la otra. "La evaluación sumativa proporciona a los maestros una idea clara de los niveles de comprensión de sus alumnos. Tiene lugar al final del proceso de enseñanza y aprendizaje y permite a los alumnos demostrar lo que han aprendido. Puede abarcar varios aspectos simultáneamente: ofrece información sobre el aprendizaje del alumno y el proceso de enseñanza que permite mejorar ambos, mide la comprensión de la idea central y lleva a los alumnos a actuar. La evaluación formativa brinda información que se utiliza para planificar la siguiente etapa del proceso de aprendizaje. Está inextricablemente unida al aprendizaje y ayuda a maestros y alumnos a identificar lo que los alumnos ya saben y son capaces de hacer. Se relaciona directamente con la enseñanza y ambos procesos se combinan perfectamente para lograr los objetivos propuestos. Tiene como objetivo fomentar el aprendizaje mediante la información frecuente y periódica sobre los resultados obtenidos, lo cual contribuye a que los alumnos amplíen sus conocimientos y comprensión, se sientan estimulados a seguir aprendiendo, reflexionen, desarrollen la capacidad de autoevaluarse y reconozcan los criterios con los que se miden los logros. Se ha constatado que un mayor uso de la evaluación formativa ayuda de manera especial a los alumnos de bajo desempeño a mejorar significativamente." Según lo mencionado anteriormente que se describe en el programa curricular de evaluación en el programa de escuela primaria, se evidencia que estas reglas se ven claramente en los procesos a evaluar, integrando la comprensión, reflexión, dedicación e interés por lograr un aprendizaje significativo.

Por otra parte se encontró, que en la otra institución son también usados los exámenes pero de diferente manera, los cuales son únicamente de carácter cuantitativo y se realizan cada dos meses donde las niñas/os deben responder ciertas preguntas sobre lo que se vio de inglés durante el bimestre, la presentación de los mismos implica que los estudiantes memoricen los temas vistos para poder responder las 
preguntas; ésto sirven para medir el conocimiento y pasarlo a una nota para rendirle informe a los papás acerca de cómo va el estudiante en la materia, limitándose únicamente a una nota.

Por otra parte, se evidencia que la institución utiliza varios instrumentos y estrategias de evaluación de forma cualitativa y cuantitativa, los cuales permiten tener evidencias del aprendizaje de los niños/as, uno de ellos emplean sólo la memorización por parte de los estudiantes, pero estos exámenes solo se aplican una vez al bimestre, teniendo en cuenta que los niños/as no están en contacto con la lengua seguido sino solamente cuatro horas a la semana, lo que implica que utilicen la memoria como instrumento para responder los exámenes, de igual forma, están las exposiciones y trabajo en equipo que le permite a los niños desarrollarse en otro tipo de ambiente y conocer la lengua que se está aprendiendo siendo evaluados de forma cuantitativa, permitiendoles reflexionar y mejorar sus aprendizajes.

De igual manera, contamos con las vivencias $y$ observaciones realizadas en las instituciones, detectando una gran diferencia la una con la otra, ya que una solo cuenta con cuatro horas de inglés a la semana; el estudio se basó en los instrumentos de evaluación propuestos por Frida Díaz (2005) y Dorothy Spiller (2011), con los cuales se caracterizaron los Instrumentos de evaluación utilizados por las maestras para evaluar el inglés.

Teniendo en cuenta la caracterización anteriormente nombrada, se encuentran unas convergencias y divergencias que dan a entender, cuál de los instrumentos y estrategias propician el aprendizaje significativo de la lengua extranjera inglés en niños y niñas de 5 a 8 años, dando cuenta de la coherencia entre lo que dicen los documentos legales y lo que realmente se evidenció, demostrando que la institución número uno cuenta con la variedad de instrumentos mencionados, los cuales proporcionan un aprendizaje significativo, pues es un proceso cíclico y constante que permite que el estudiante esté en todo momento interesado por aprender algo nuevo del otro idioma; además se ve a la evaluación dentro del PEP con el "objetivo de guiar a los alumnos cuidadosa y eficazmente a través de los cinco elementos esenciales del aprendizaje: la adquisición de conocimientos, la comprensión de conceptos, el dominio de habilidades, el desarrollo de actitudes y la decisión de actuar. El objetivo principal de la evaluación en el PEP es el de proporcionar información acerca de la marcha del proceso de aprendizaje. 
Análisis de las estrategias e instrumentos de evaluación en el aprendizaje del inglés como lengua extranjera

Se espera que todos los colegios desarrollen procedimientos de evaluación y métodos de información acerca de ésta que estén condensados en la filosofía y los objetivos del programa" reflejadose en el actuar, hablar y pensar de los niños.

Esta investigación en la institución no solo tuvo unos cuantos meses, sino que fue un proceso a lo largo de un año, en el que las prácticas permitieron estar inmersos en la vida cotidiana desde una perspectiva didáctica, evidenciado los espacios de evaluación que propiciaban y fomentaban un mayor aprendizaje en los niños y las niñas.

Una diferencia encontrada en las instituciones está en que cada una de ellas maneja el rango de edad de manera distinta, en una en el grado de transición se encuentran los niños de 5 años y en la otra los 7 años, por eso en esta última el nivel de exigencia y aprendizaje es aún mayor, teniendo en cuenta que la inmersión al idioma, es un proceso, no se hace todo al tiempo, evitando que se genere confusión entre la lengua materna y la lengua inglesa. De esta manera hasta los 7 años cuando ya está afianzada su escritura, pueden tener la posibilidad de practicar la escritura en el idioma inglés, antes de esta etapa, solo se propician temas de lenguaje $y$ escucha.

\section{Conclusiones}

Esta investigación tuvo como objetivo caracterizar las estrategias de enseñanza e instrumentos de evaluación utilizados con los niños y niñas entre 5 y 8 años de edad, de dos instituciones educativas de la ciudad de Bogotá, para reconocer cuáles de ellos propician el aprendizaje del inglés como lengua extranjera; de esta manera, conocer cuáles de estos instrumentos y estrategias de evaluación utilizan las maestras para la enseñanza del inglés, que realmente propicien y motiven el aprendizaje de la lengua extranjera en los niños y las niñas.

De acuerdo con lo anterior, los resultados obtenidos de la investigación, teniendo en cuenta la observación y el análisis de las instituciones se puede ver que en la institución número uno Gimnasio del Norte ven la evaluación como algo constante, debido a que en ella está la formación integral del niño. Se evidencia que toman ésta como un proceso constante y cíclico, lo cual da cuenta de que realmente "La evaluación debe ser recurso de formación y oportunidad de aprendizaje, tanto para los maestros como para los estudiantes, debido a que es de esta manera que es integral y comprensiva, además, para la evaluación se debe tener en 
cuenta la formación del niño, y saber que éste no es una página en blanco; conociendo sus intereses, habilidades y procesos de aprendizaje" (Meirieu, 1998). Además, la evaluación la ven como una herramienta de aprendizaje pues es utilizada en todo momento y de esta manera pueden observar los aprendizajes de los niños y niñas; teniendo en cuenta que ellos más adelante tendrán la oportunidad de hacer intercambios y conocer nuevas culturas. Este aprendizaje del idioma es guiado según de las unidades de indagación, enseñando para la vida, se explora el medio y no únicamente llegan a que quieran pasar un examen.

Por otro lado, según los resultados de la investigación, se puede observar que la institución número dos Colegio la Presentación Fátima ve la evaluación de forma cualitativa y cuantitativa, resaltando la importancia de que la evaluación es necesaria, vista desde la autoevaluación y coevaluación, puesto que en las jornadas de evaluación se hacen actividades donde los niños aprenden a autoevaluarse, utilizando diversos instrumentos de evaluación como el examen, quices, trabajos e informes. Las maestras a pesar de que ven la parte cualitativa del proceso, cada uno de estos instrumentos es utilizado de manera cuantitativa; debido a que los estudiantes tienen únicamente cuatro horas a la semana en las que pueden estar inmersas en la lengua, lo que genera que muchas veces los aprendizajes sean dirigidas únicamente en torno a la calificación.

Teniendo en cuenta lo anterior, ambas instituciones dan cuenta de que la evaluación es un proceso de aprendizaje, como lo menciona Álvarez Méndez (2001) siendo ésta una actividad crítica del aprendizaje, porque se asume que gracias a ésta adquirimos un mayor conocimiento; se trata de buscar la manera de que sea crítica de tal forma que los maestros se preocupan por utilizar diversos instrumentos de evaluación que propicien el aprendizaje entre los cuales están: los trabajos grupales, portafolios, quices, presentaciones, informes, entre otros, llegando a ser integrados de manera que se tenga en cuenta la forma cualitativa y cuantitativa.

De igual manera las maestras se interesan porque se genere un interés y se disfruten las clases, poniendo a prueba la imaginación y creatividad en las exposiciones orales, dando cuenta que estas también les propician cierto aprendizaje, sin embargo, aunque conocen algunos temas de la lengua extranjera, se les dificulta realizar oraciones y comprender cuando la profesora les da las instrucciones en inglés. Teniendo en cuenta las entrevistas realizadas, se encontró que en ocasiones los padres de familia se 
Análisis de las estrategias e instrumentos de evaluación en el aprendizaje del inglés como lengua extranjera

interesan por practicar en casa con sus hijos los temas vistos, de tal manera que se preparen aún más para los exámenes bimestrales.

Así mismo, se evidencia que aunque en los colegios se vea la materia de inglés, su aprendizaje no es significativo, sino que se preparan en temas básicos, con poco lenguaje y conocimiento del mismo. Es importante recalcar que Colombia es el país con el menor porcentaje en América Latina con el 45,1\%, según lo establecido por el English Proficiency Index en el 2012, asimismo, se tiene en cuenta que hoy en día el tener conocimiento de una sola lengua no es suficiente en un mundo interconectado. Considerando el porcentaje anteriormente mencionado, es importante que en el país se implemente una intensidad horaria mayor, con profesionales preparados para que se incentive y despierte el interés por aprender una nueva lengua, teniendo la oportunidad de conocer otra cultura y costumbres. Notablemente se evidencia que la formación para la vida es la manera en la que el aprendizaje es significativo, dando valor a lo que se está aprendiendo.

A pesar de que no se han encontrado antecedentes de esta investigación, es importante seguir realizando investigaciones de este tipo porque en el aprendizaje de una lengua no es solo importante poder comprender diferentes temas básicos, sino que el estar inmerso en todo lo que conlleva aprender de manera adecuada un lenguaje nuevo, permite estar interconectado en el mundo, establecer relaciones sociales con diferentes personas, conocer $y$ enriquecer las propias culturas desde otro punto de vista.

\section{Referencias}

Barriga, F. D. (2005). La evaluación auténtica centrada en el desempeño: Una alternativa para evaluar el aprendizaje y la enseñanza. En Enseñanza situada: Vínculo entre la escuela y la vida. México: McGraw Hill.

COLEGIO DE LA PRESENTACIÓN DE FÁTIMA. (2015). Manual de Convivencia. Obtenido de http://www.colpresentacionfati ma.com/files/admisiones/docu mentos/MANUAL\%20DE\%20CO NVIVENCIA\%20FINAL\%202015.p df

Comenius, J. A. (1998). Didáctica Magna. Akal.

Diez, M. V. (2010). EL INGLÉS MEJOR A EDADES TEMPRANAS. $1-6$.

First, E. (2015). English Proficiency Index. Obtenido de http://media2.ef.com/__/ /med ia/centralefcom/epi/downloads /full-reports/v5/ef-epi-2015spanish.pdf 
Gimnasio del Norte. (2007). LA EVALUACIÓN EN EL PEP. Obtenido de IBO Programa de la escuela Primaria, "Cómo hacer realidad el PEP": http://www.gimnasiodelnorte. edu.co/documentos/document os/la-evaluacion-en-el-PEPespaniol.pdf

Kant, I. (1986). Pedagogía. Akal.

Lambert, W. E. (1987). Cuestiones sobre la lengua extranjera y la enseñanza de la segunda lengua. Obtenido de Contro Virtual Cervantes: http://cvc.cervantes.es/ensena nza/biblioteca_ele/antologia_d idactica/inmigracion/lambert. htm

Locke, J. (1986). Pensamientos sobre la educación. Madrid: Akal.

Meirieu, P. (1998). Frankestein Educador. Laertes.

Mejía, A.-M. d. (2011). The National Bilingual Programme in Colombia: Imposition or opportunity? Apples - Journal of Applied Language Studies, 1-11.

Mejía, A.-M. T. (2009). Orientaciones para políticas bilingües y. Centro de Investigación y Formación en Educación.

Méndez, J. M. (2001). Evaluar para conocer, examinar para excluir. Madrid: Morata.

Piñeros, L. (19 de 04 de 2003). La llave: estándares, evaluación, mejoramiento. Obtenido de $\mathrm{Al}$ Tablero:

http://www.mineducacion.gov. co/1621/article-87448.html

RÍOS, L. M. (19 de 02 de 2014). Ser bilingüe aporta valor competitivo. El Colombiano.

Saavedra, L. S. (s.f.). Entre lo deseable $y$ lo realizable: didáctica, currúculo y evaluación. currículo evaluación y criterios: pedagogía para la autonomía y la democracia, 35-53.

Silva, A. R. (s.f.). Texto, Testimonio y Metatexto. En La Práctica investigativa en Ciencias Sociales (págs. 45-61). Bogotá.

Spiller, D. (2011). Assessment tasks to promote learning. Teaching Development.

Zuluaga, O. L. (2003). Pedagogía y Epistemología. Magisterio. 


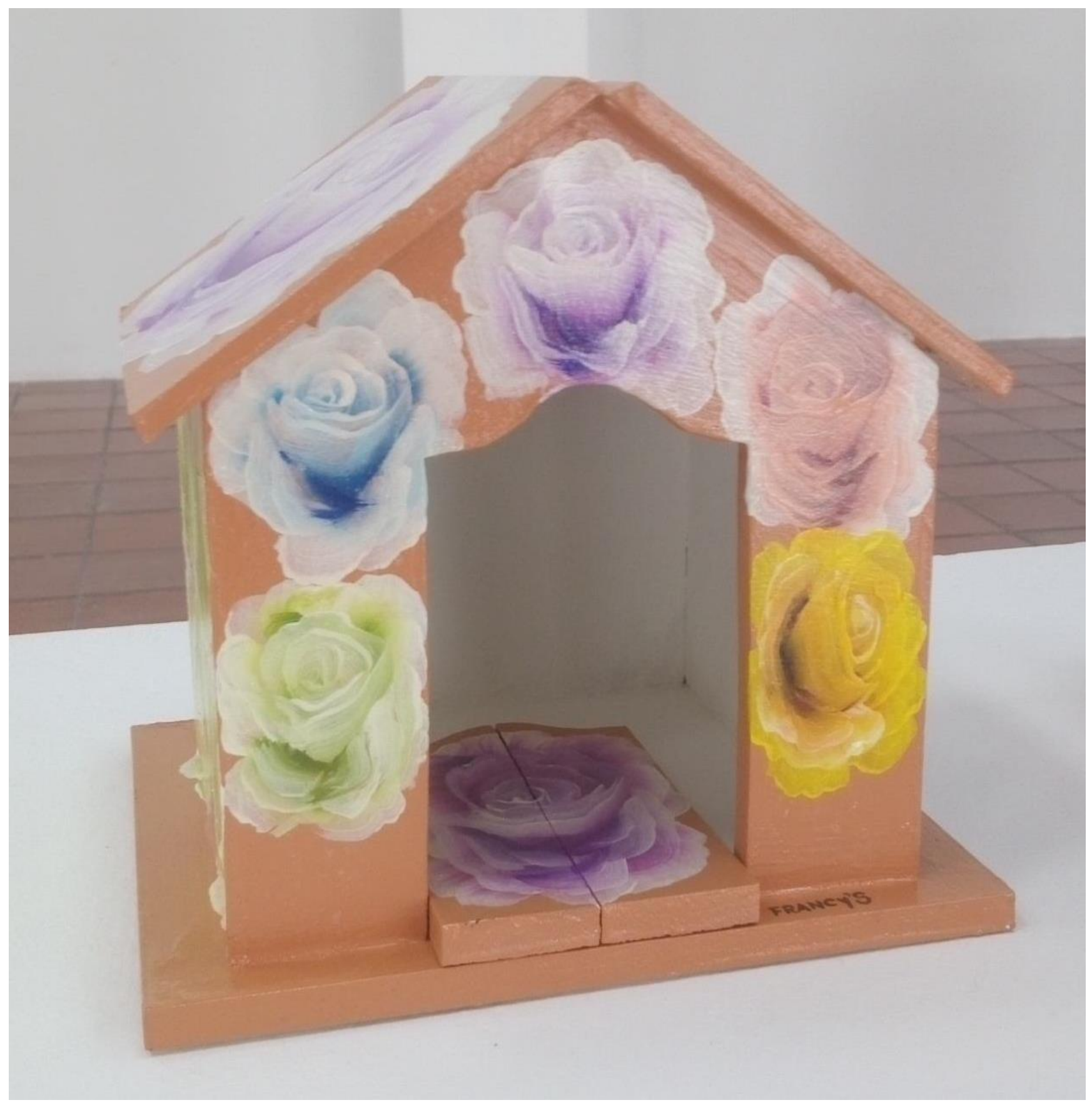

Obra: Que florezca la paz

Artista: Francy Elena Conde Técnica: Acrílico sobre madera Territorio: Neiva 